\title{
Metallic Nanofilm Enhanced Fluorescence Cell Imaging: A Study of Distance-Dependent Intensity and Lifetime by Optical Sectioning Microscopy
}

Yan-Yun Zhai, Qian Liu, Wei-Peng Cai, Shuo-Hui Cao, Li-Xiang Zhang, Yao-Qun Li*

Department of Chemistry and the MOE Key Laboratory of Spectrochemical Analysis \& Instrumentation, College of Chemistry and Chemical Engineering, Xiamen University, Xiamen 361005, China

\section{SUPPORTING INFORMATION}

* Corresponding author: Yao-Qun Li, Tel./Fax: +86-592-2185875.

E-mail: yaoqunli@xmu.edu.cn. 

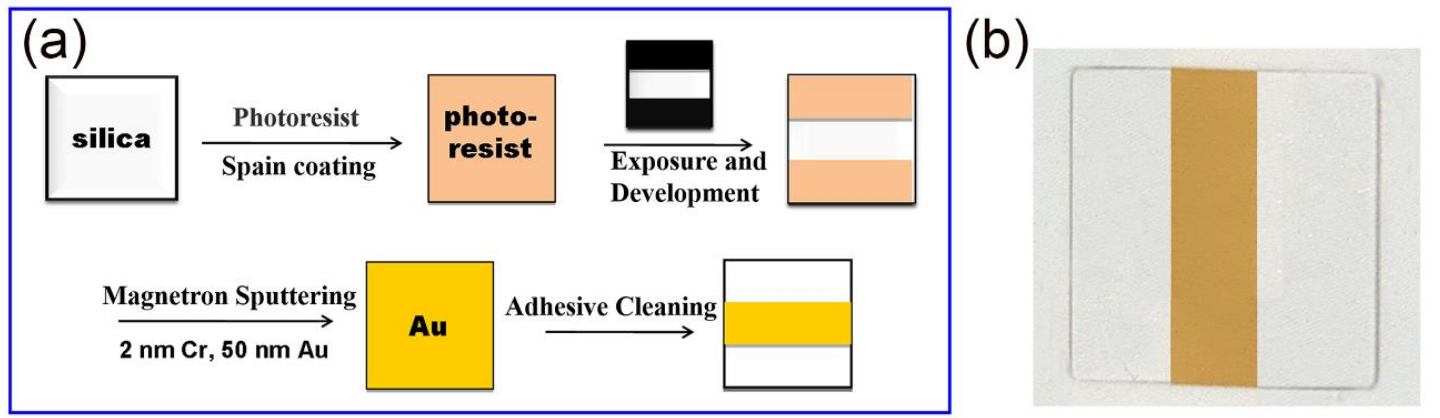

Figure S1. (a) The preparation procedure of the gold film stripe on the quartz substrate and (b) the picture of the stripped gold substrate.

The preparation procedure of the gold stripe on the quartz substrate:

(1) Spin coating and pre-baking: The clean quartz substrate was activated in an oven at $135^{\circ} \mathrm{C}$ for $1 \mathrm{~h}$. The BP212 was span coating on the surface of the activated quartz substrate at a speed of $2000 \mathrm{rpm}$. Then the substrate was baked in a vacuum oven at $110{ }^{\circ} \mathrm{C}$ for $15 \mathrm{~min}$. The purpose of the prebaking is to evaporate the solvent of the photoresist to increase adhesion.

(2) Exposure, development and post-baking: The BP212 positive gel was exposed by using the film shown in Figure S1a as a photolithography mask. With a $0.5 \% \mathrm{NaOH}$ solution as the developer and developing time of $28 \mathrm{~s}$, the photoresist in the middle of the quartz substrate fell off. The developed substrate was baked in a vacuum oven at $100{ }^{\circ} \mathrm{C}$ for $15 \mathrm{~min}$. The purpose of post-baking is to evaporate the residual developer and the cleaning solution and to make the polymer structure in the photoresist more compact to reduce photoresist defects, improve etching resistance, and increase flatness.

(3) Magnetron sputtering: A $2 \mathrm{~nm}$ adhesive layer of chromium followed by a $50 \mathrm{~nm}$ gold continuous film was sequentially deposited onto the above substrate via radiofrequency 
magnetron sputtering.

(4) Degumming: The substrate after magnetron sputtering was immersed in acetone overnight. The gold film on the surface of the photoresist was detached with the dissolution of the photoresist by ultrasonication, thereby obtaining a stripe-shaped Au film substrate, as shown in Figure S1b. The substrate was ultrasonically cleaned with ethanol and pure water for 10 min and dried with $\mathrm{N}_{2}$ for use.
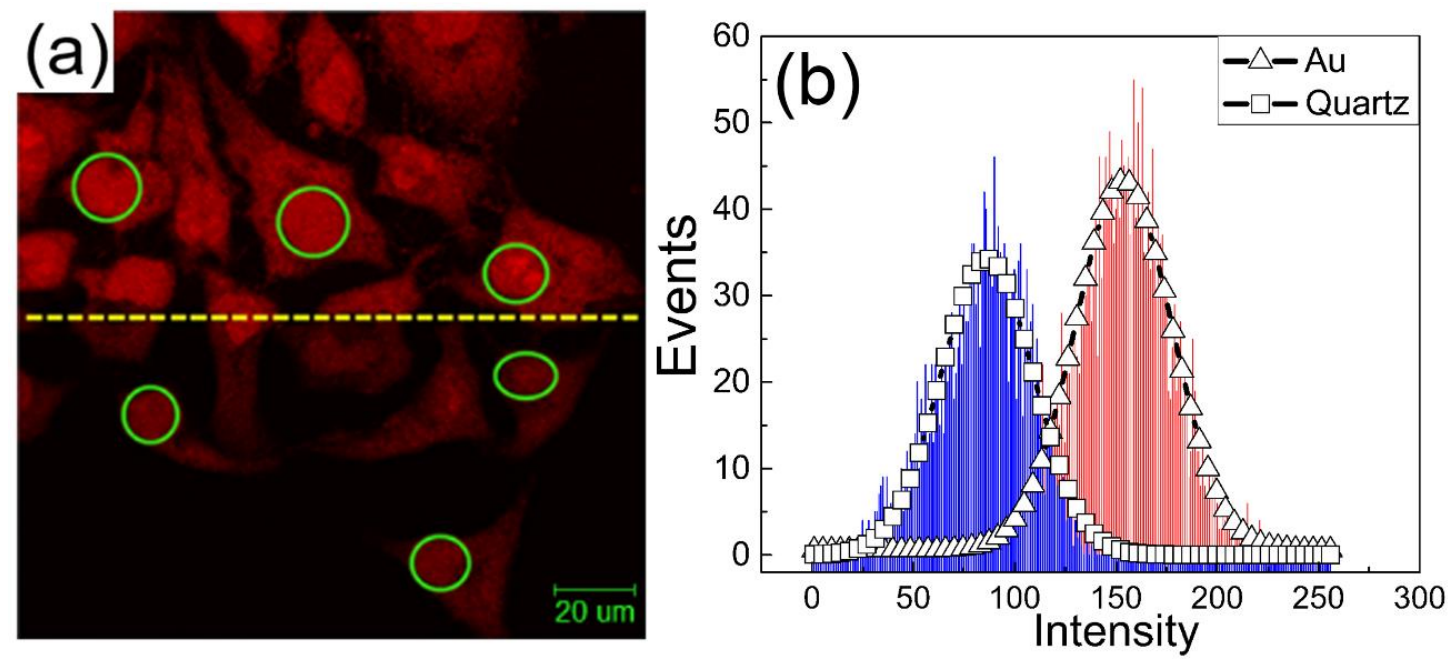

Figure S2. (a) Fluorescence image and (b) intensity distributions of PI-stained cells on Au and quartz substrates obtained by the Histogram function of Image-Pro Plus (observed by NA 1.40 objective, $\mathrm{z}=0 \mu \mathrm{m})$. The upper part is on Au nanofilm and the lower part is on the quartz substrate. 


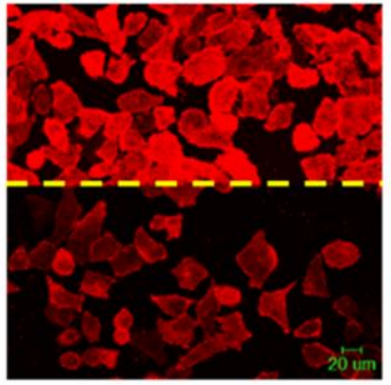

$\mathrm{z}=0 \mu \mathrm{m}$

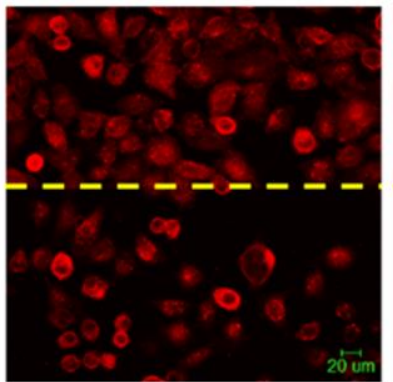

$\mathrm{z}=5 \mu \mathrm{m}$

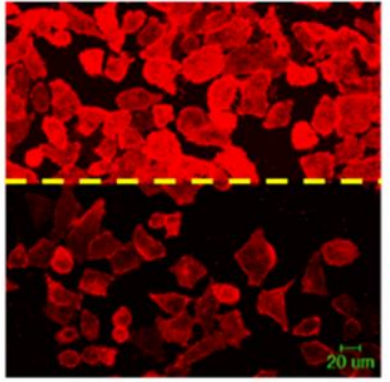

$\mathrm{z}=1 \mu \mathrm{m}$

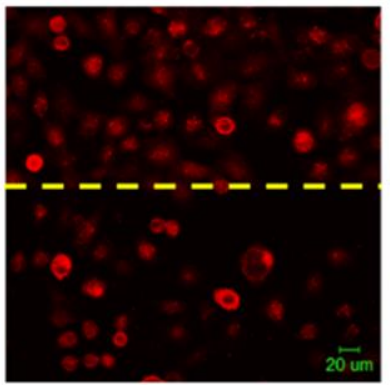

$\mathrm{z}=7 \mu \mathrm{m}$

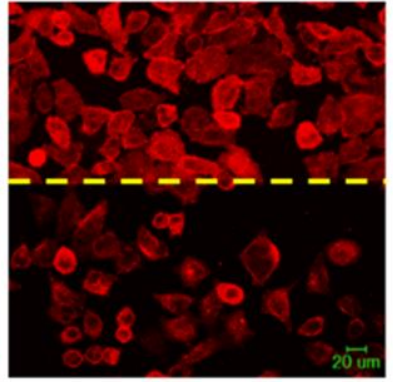

$\mathrm{z}=3 \mu \mathrm{m}$

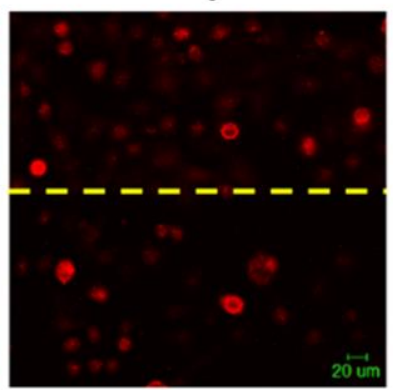

$\mathrm{z}=9 \mu \mathrm{m}$

Figure S3. Cross-section images of DiI-stained cells at various z-positions, observed by NA 0.85 objective. The upper part is on Au nanofilm and the lower part is on the quartz substrate.

The cell membrane thickness is on the order of $10 \mathrm{~nm}$ which is much smaller than the thickness of microscale cell, so theoretically the thickness of the cytoplasm is approximately equal to the thickness of the cell. The thickness of the cell was estimated by measuring the section span from the basal membrane to the upper membrane of the DiI-stained cells. We obtained a series of cross-section images of DiI-stained cells from the basal membrane to the upper membrane where the fluorescence almost disappeared, as shown in Figure S3. The section span was $9 \mu \mathrm{m}$, so we can estimate the thickness of the cytoplasm, which is approximately equal to the thickness of the cell, is about $9 \mu \mathrm{m}$. 


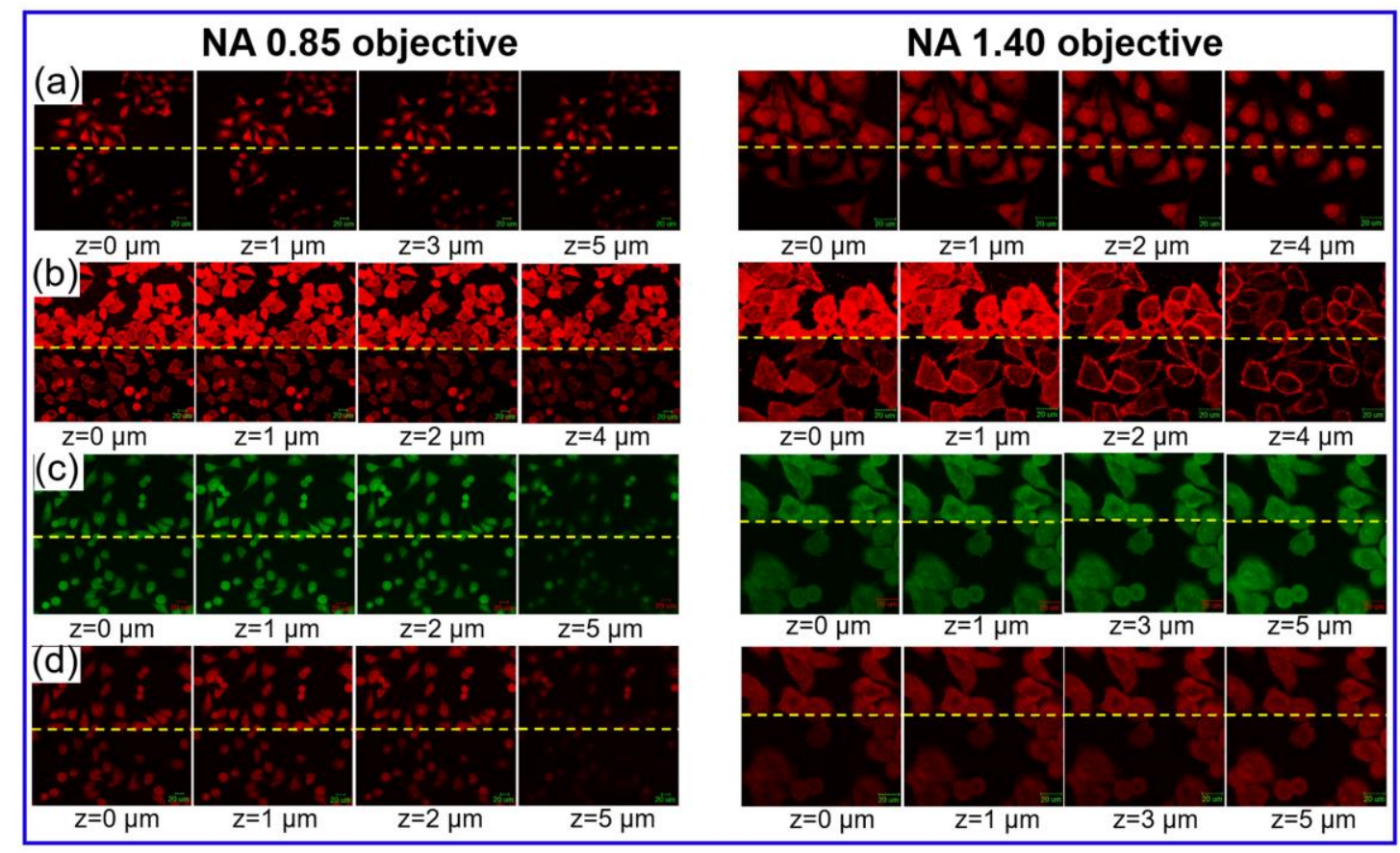

Figure S4. Cross-section images of (a) PI, (b) DiI, AO (c, AO-DNA; d, AO-RNA) stained cells at different z-positions, observed NA 0.85 objective and NA 1.40 objective. The upper part is on Au nanofilm and the lower part is on the quartz substrate.

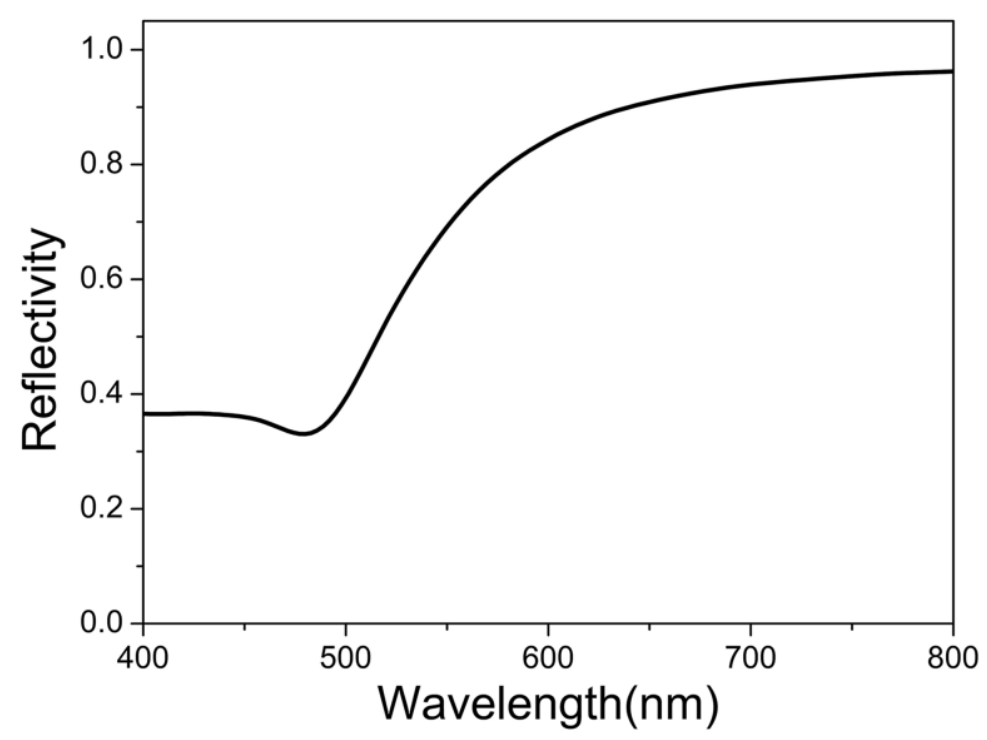

Figure S5. The simulated reflectivity curve with the wavelength of $50 \mathrm{~nm}$ Au nanofilm. 
The effect of wavelength on the enhancement factor can be described from two aspects: (1) The reflectivity of the metal film at different wavelengths are different, so the reflected field strength is different. The higher the reflectivity is, the greater the field strength is, which induce bigger enhancement factor; (2) The incident light and the reflected light form a standing wave. Consequently, the field intensity oscillates as a function of the distance from the film, alternating in-phase positions and out-of-phase positions. The strongest fluorescence enhancement factor is obtained at the in-phase positions, and the in-phase positions are related to the wavelength. That is to say, different wavelengths obtain the best enhancement effect at different positions away from the film. Conversely, the same position has different enhancement effects at different wavelengths. The interaction between these two aspects makes the metal enhancement factor and wavelength have a non-linear relationship. The former affects the strength of the enhancement factor, and the latter determines the position where to obtain the largest enhancement factor.

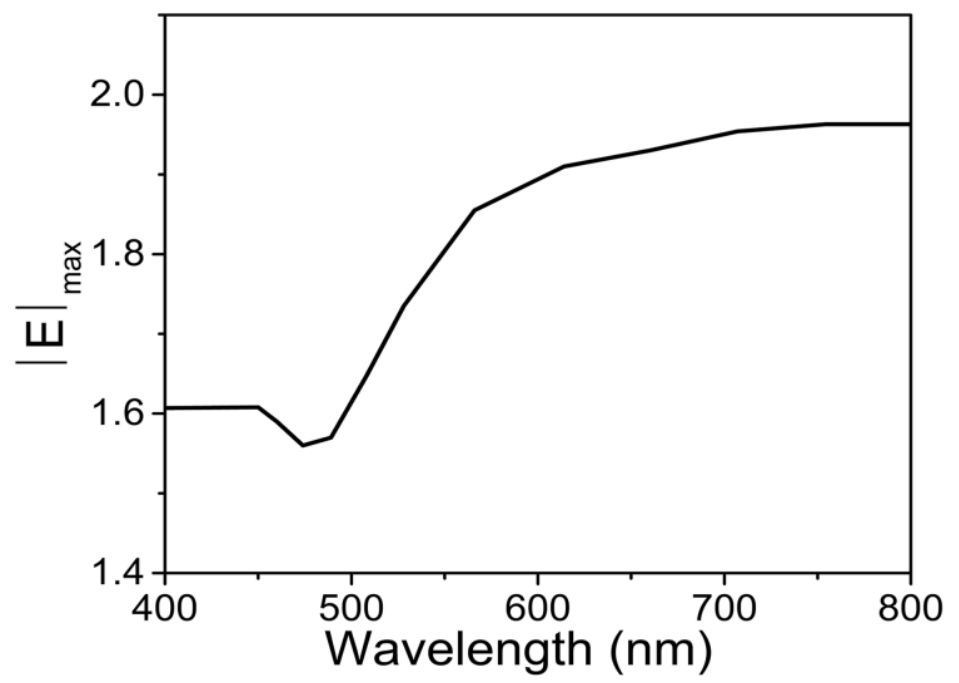

Figure S6. The calculated maximum electric field intensity for different wavelengths 

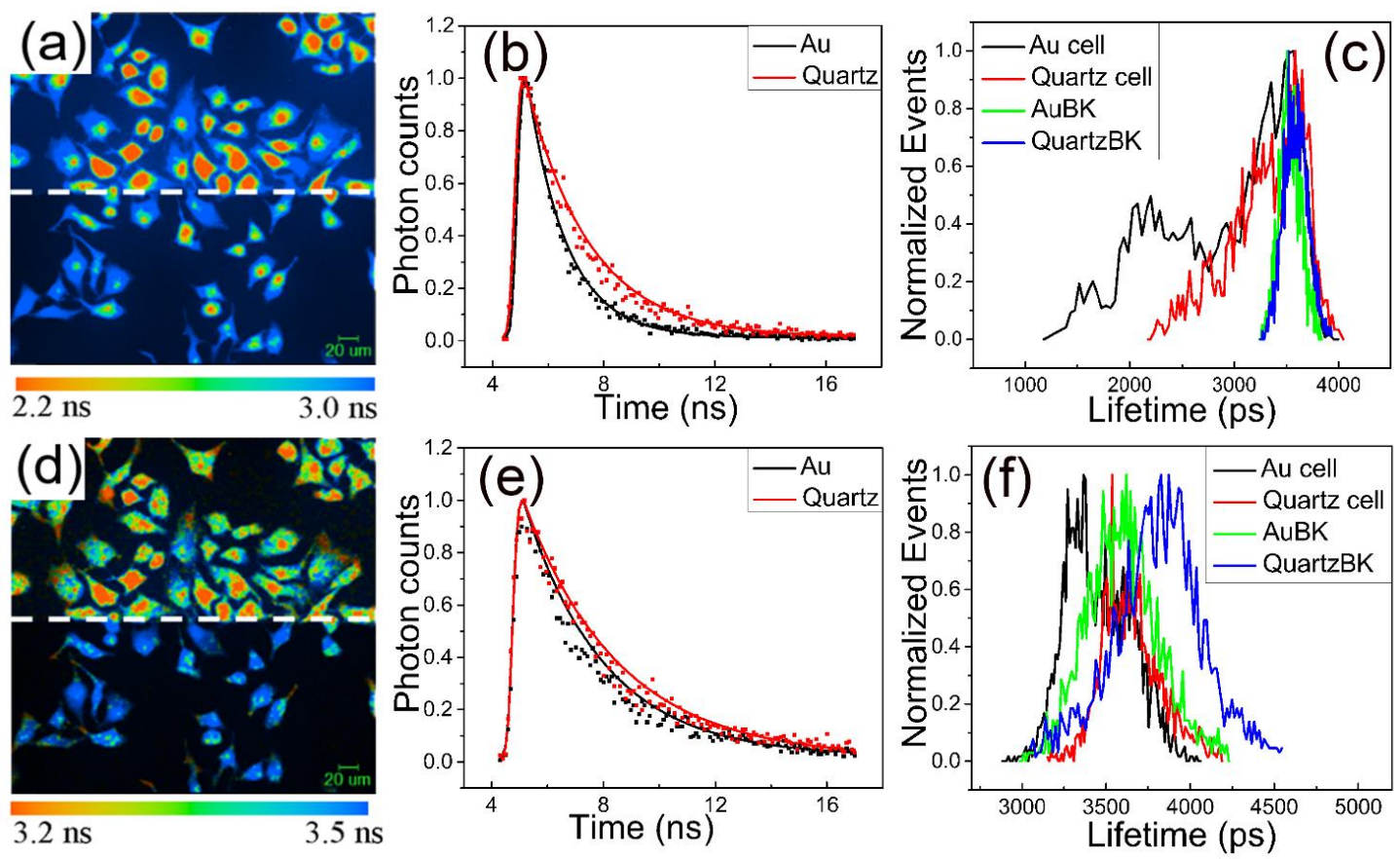

Figure S7. FLIM image, Lifetime decay curves and distributions (cell corresponding to sample,

BK corresponding to background) of AO ( $a, b$ and c, AO-DNA; d, e and f, AO-RNA) stained cells on $\mathrm{Au}$ and quartz substrates, obtained by NA 0.85 objective. The upper part is on Au nanofilm and the lower part is on the quartz substrate. 

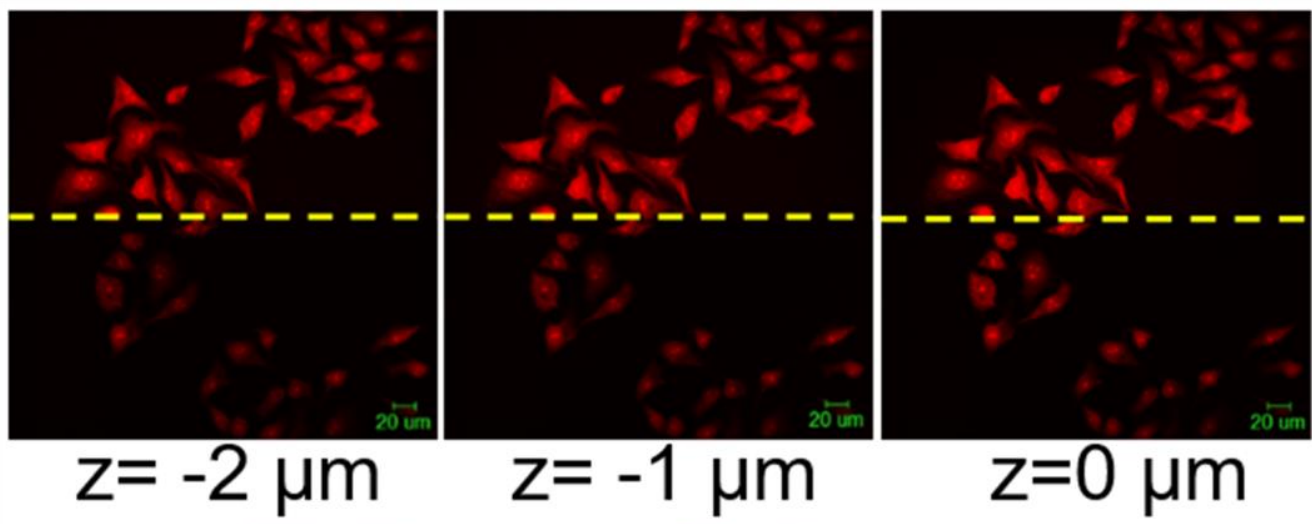

$\mathrm{z}=0 \mu \mathrm{m}$
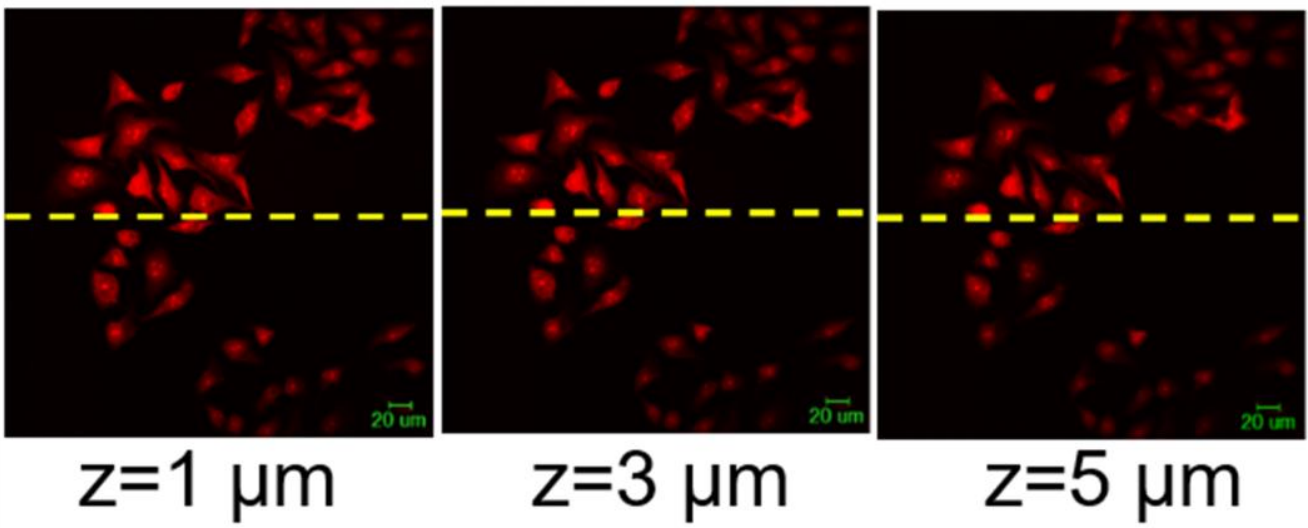

Figure S8. Cross-section images of PI-stained cells at various z-positions $(\mathrm{z}<0 \mu \mathrm{m}$, defined as the substrate side; $\mathrm{z}=0 \mu \mathrm{m}$, defined as the interface; $\mathrm{z}>0 \mu \mathrm{m}$, defined as the cell side), observed by NA 0.85 objective. The upper part is on Au nanofilm and the lower part is on the quartz substrate.

The position when the focal plane reached into the substrate was defined as the substrate side. When the substrate can reflect the illuminated light, the image of the sample can be observed at the substrate side. Figure S8 shows a series of cross-section fluorescence images of the cells at different $\mathrm{z}$ positions. In addition to the images got at the interface and at the cell side, the clear images of the cells can also be observed at the substrate side for both Au film and quartz substrate, which was generated by the reflection of the substrate. The gold film has a high reflectivity inducing a strong mirror effect, so the corresponding fluorescence intensity of the 
images at Au film substrate side is stronger. The intensity of the cell images is weaker at the quartz substrate side because of its lower reflectance. Therefore, the fluorescence lifetime at the interface for the quartz sample is also slightly shortened due to its weak mirror effect.

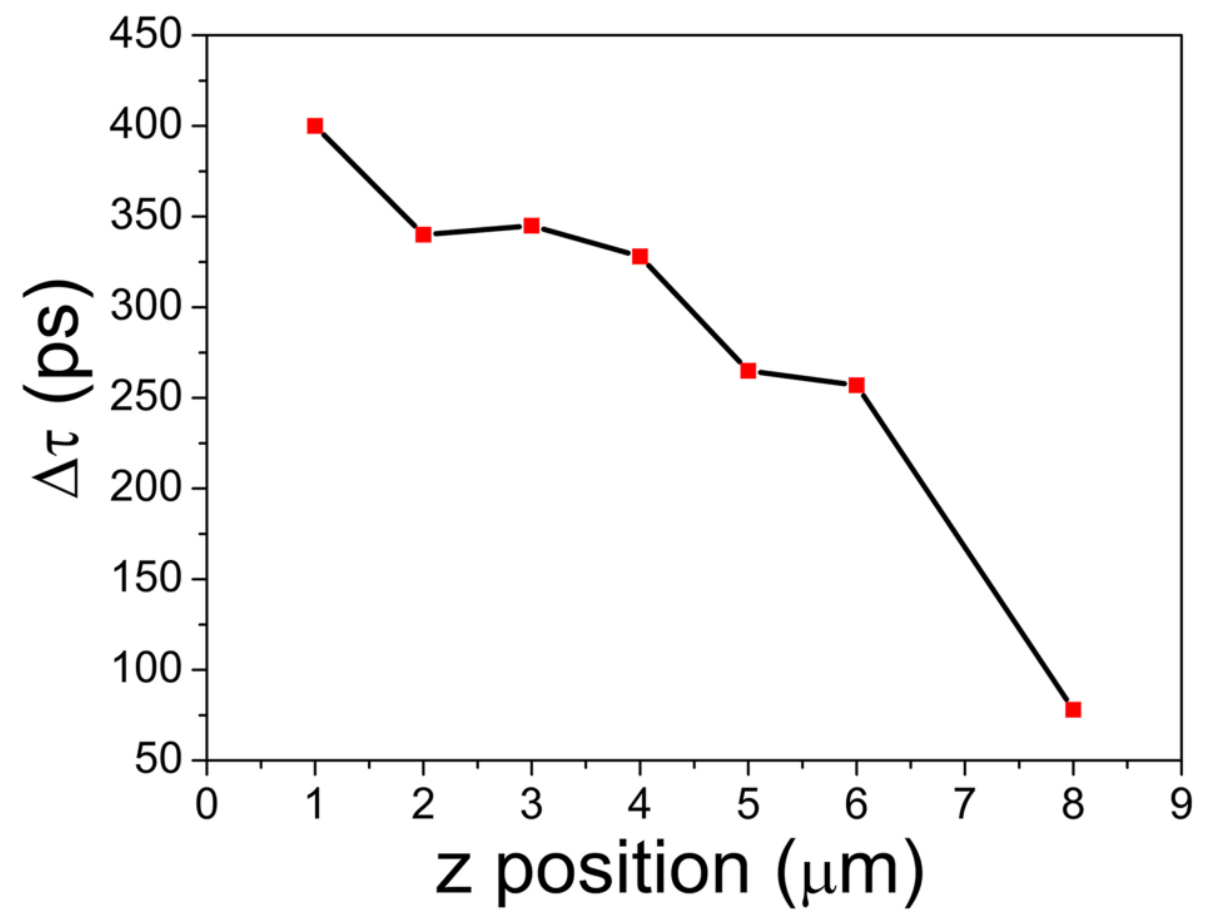

Figure S9. The $\Delta \tau$ of PI-stained cells observed by NA 0.85 objective at different z-position for another location. 

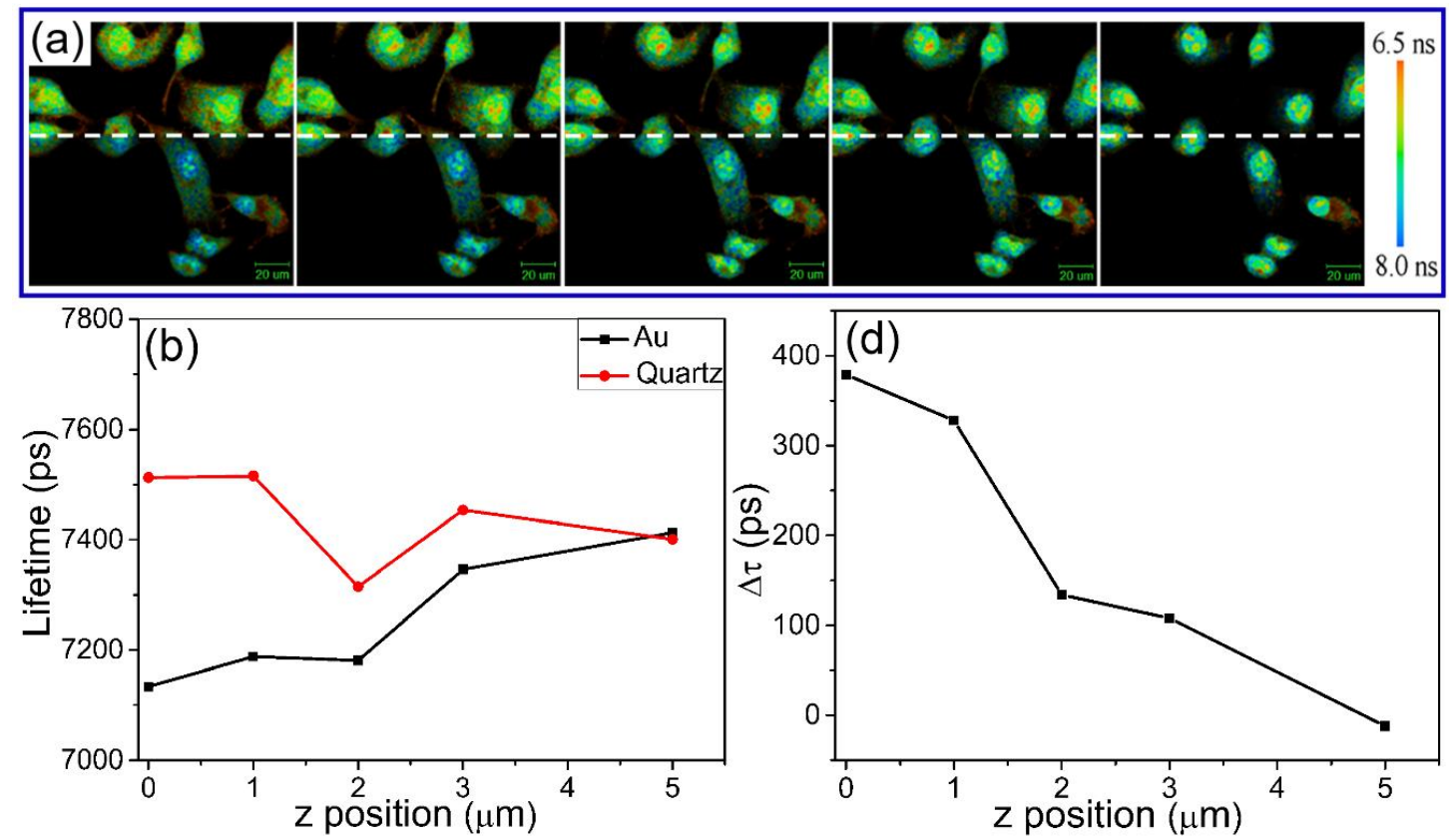

Figure S10. (a) Cross-section FLIM images, (b) Fluorescence lifetime and (c) $\Delta \tau$ of PI-stained cells observed by NA 1.40 objective at different z-position. The upper part is on Au nanofilm and the lower part is on the quartz substrate. 